\title{
The number of letters in number words influences the response time in numerical comparison tasks: Evidence using Korean number words
}

\author{
Doyeon Kwon ${ }^{1}$ - Songjoo Oh ${ }^{1}$
}

Published online: 11 October 2019

(C) The Psychonomic Society, Inc. 2019

\begin{abstract}
Here, we report that the number of letters in number words influences the response time in numerical comparison tasks. In this experiment, a pair of single Korean number words consisting of two or three letters was simultaneously presented in an area of the same size, and the participants reported which was semantically larger. The conditions were categorized as congruent, neutral, and incongruent based on the congruency between the meaning indicated by the numeral (i.e., the size of the number or semantic size) and the number of letters in each number word. In the analysis, compared to the neutral (faster) and incongruent (slowest) conditions, the response time was the fastest under the congruent condition. Thus, the congruency effect is explained by the number of letters rather than continuous visual properties (occupied area and length). These results suggest that the semantic representation of number words is automatically influenced by the number of letters they contain.
\end{abstract}

Keywords Size congruency effect · Numerical Stroop effect $\cdot$ Korean number words · Number of letters

\section{Introduction}

In a numerical comparison task, the response time (RT) varies depending on the physical size of the number notations even though this size is irrelevant to the task. Typically, when the semantic and physical dimensions of the number notations being compared are congruent, the RT is faster than that when the dimensions are not congruent (Besner \& Coltheart, 1979). This size congruency effect has been extensively tested in a variety of number notations, including Arabic numerals (Henik \& Tzelgov, 1982), English scripts (Damian, 2004; Foltz, Poltrock, \& Potts, 1984; Vaid, 1985), Chinese scripts (Tzeng \& Wang, 1983), Hebrew scripts (Razpurker-Apfeld \& Koriat, 2006), and Japanese Kanji scripts (Ito \& Hatta, 2003; Takahashi \& Green, 1983). This effect clearly shows that the semantic processing of numbers is not independent of the visual properties, such as the physical size, occupied area, and length, although the level of numerical processing at

Songjoo Oh

songjoo@snu.ac.kr

1 Department of Psychology, College of Social Sciences, Seoul National University, 1 Gwanak-ro, Gwanak-gu, Seoul 08826, South Korea which the interaction occurs remains debatable, i.e., whether the interaction occurs at an early interactive representation stage (Schwarz \& Heinze, 1998) or a late competitive decision stage (Cohen Kadosh, Gevers, \& Notebaert, 2011; Sobel, Puri, Faulkenberry, \& Dague, 2017).

In this study, we attempt to expand the size congruency effect from a continuous dimension to a discrete dimension (hereafter, for the convenience of discrimination, we call these effects the continuous size congruency effect and the discrete size congruency effect, respectively). In general, printed number notations are composed of several figural parts, including strokes, bars, circles, and squares, and these parts appear alone in connected forms, such as Arabic digits, or disconnected forms, such as English number words. Therefore, does the number of such parts influence the RT in numerical comparisons? The answer to this question, considering the current dominant theories of numerical representation could be interesting. Furthermore, it has been extensively suggested that the discrete and continuous properties of objects serve as the core system of numerical representation (Feigenson, Dehaene, \& Spelke, 2004; Henik, Leibovich, Naparstek, Diesendruck, \& Rubinsten, 2012; Leibovich \& Henik, 2013). Here, the discrete properties are defined by countable quantities, such as the number of individual objects, while the continuous properties are defined by uncountable quantities, such as the font 
size, occupied area, and length. Education may combine these two concepts into symbolic systems, such as Arabic numerals or number words. From this perspective, the continuous size congruency effect implies that the semantic representation of number notations is never free from the processing of the continuous properties of notations, even though learning renders such properties relative abstractions.

Considering that the discrete properties of number notations are extensively described in the literature and frequently experienced in daily life, why is it difficult to find studies showing that the number of parts in number notations influences the RT in numerical comparisons? One likely reason may be the inherent methodological limitations of these number notations. Specifically, the discrete properties of number notations largely change along with the continuous properties; thus, it is difficult to isolate the pure effect of the first from that of the second (see Leibovich \& Henik, 2013; Mix, Huttenlocher, \& Levine, 2002, for reviews). First, all single Arabic digits (1-9) have parts or strokes that are connected into one shape; thus, it is difficult to manipulate their discrete properties. Hindi, Indian, and Hebrew number words have limitations similar to those of Arabic digits because the parts or strokes of these number words are also all connected into one shape. Second, the English number words (one-nine) popularly used to test the size congruency effect have different properties that are problematic in demonstrating the discrete size congruency effect because changes in the number of letters inevitably lead to changes in the size of other continuous properties, such as the area occupied by the letters (Dixon, 1978; Dormal, Seron, \& Pesenti, 2006). Previous studies controlled for the discrete properties of number notations such that the same number was used across conditions. For example, the pair of alphabetic numerals presented had the same number of letters, e.g., one-six, four-five, or threeseven (Besner \& Coltheart, 1979; Foltz et al., 1984). The primary interest of these studies was to observe the pure effect of continuous properties, such as physical size, on numerical representation. Thus, it is understandable that the investigators in these studies used word stimuli consisting of the same number of letters.

Nevertheless, we argue that in addition to continuous properties, providing evidence supporting the effect of discrete properties on numerical comparisons is crucial because such evidence could help reveal their core role in numerical representation. Based on this perspective, Korean number words are a convenient tool to address this question because they comprise several letters and are conventionally written within the same physical area; therefore, the problem of the mixed effect of continuous and discrete properties can be avoided. Thus, the purpose of this study was to use Korean number words to investigate the effect of the number of letters in a single word on numerical comparison tasks.

The Korean writing system, which is called Hangul, consists of 24 letters: 14 consonants and 10 vowels. Specifically,
Korean number words representing single-digit numbers (from 1 to 9) consist of one vowel + one or two consonant(s). For example, as shown in Fig. 1, “삼”, which means "three", is composed of three letters as follows: two consonants “人” and "ロ" and one vowel "ト". More importantly, the letters constituting the number words are placed within an imagery square without any connection; thus, the occupied areas and lengths are approximately the same across all single-digit number words. The number of letters that constitute each number word is not related to the meaning indicated by the word (i.e., the semantic size of the number); thus, when two number words are compared, the number of letters and the semantic size can be incongruent across two number words. For example, "오", i.e., "five", is composed of two letters, i.e.,

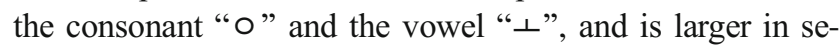
mantic size than “삼", i.e., "three", but contains fewer letters (two vs. three). Therefore, Korean number words are appropriate stimuli for examining the effect of the number of letters on RT in a numerical comparison task while controlling for the size of the occupied area. In this study, we hypothesized that if the number of letters in Korean number words was automatically activated, when the semantic size of the number and the number of letters were congruent, the RT could be faster than that when they were incongruent.

For comparison with the Korean number word condition, an Arabic digit condition was also introduced. However, in studies using a Stroop-like numerical task, Arabic digits have been widely examined in other countries, but such studies involving Korean people are rarely documented. Thus, the data obtained under the Arabic condition are expected to provide an objective reference for estimating the data obtained under the Korean condition. Korean scripts, including number words, are phonologically transparent. For example, each letter has its own phoneme, and this matching is highly regular. Arabic digits and the corresponding Korean number words have the same pronunciation and the same meaning. Thus, regarding our hypothesized congruency effect, we aimed to confirm that this effect is due to the visual factors of the stimuli and not to any potential differences involving either phonological or purely semantic factors. In addition to the size congruency effect, to ensure that the semantic sizes of the stimuli were activated for both the Arabic and Korean notations, subsequent tests were conducted to determine the presence of distance effects under each notation condition (Dehaene \& Akhavein, 1995).

\section{Methods}

\section{Participants}

Twenty-six college students (nine females and 17 males, mean age $=20.3, S D=1.87$ ) participated in the study. This sample size was determined by performing a power analysis based on 

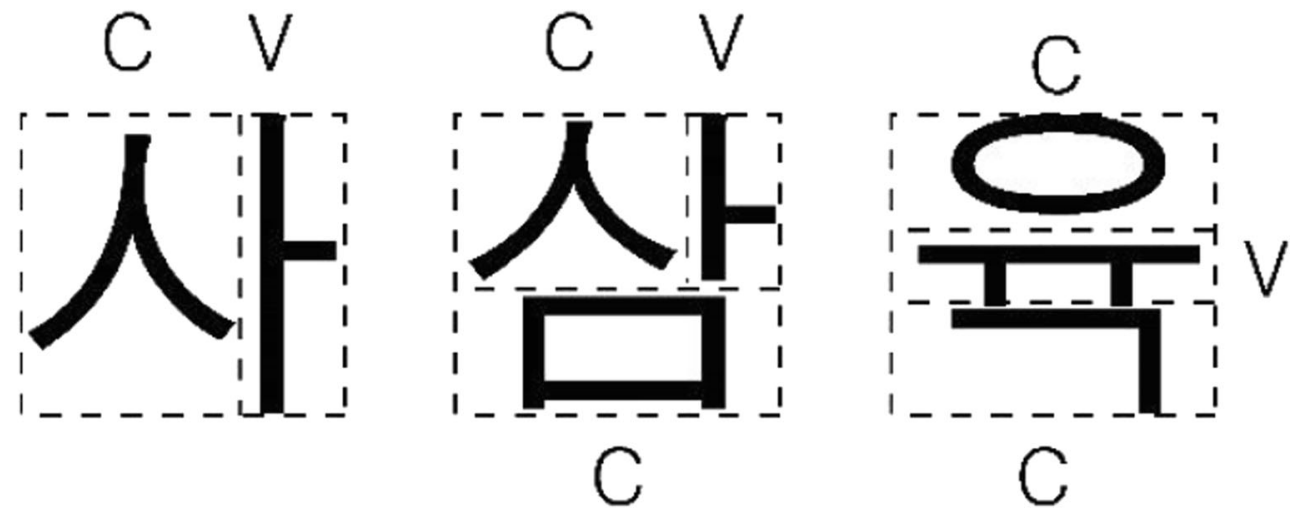

Fig. 1 Examples of Korean number words. C indicates a consonant, and V indicates a vowel (number words corresponding to the Arabic digits 4, 3, and 6)

the size congruency effect of English number words in the study conducted by Vaid (1985; paired samples $t$-test; $d_{z}=$ 0.59 ; power $=.80, \alpha=.05$, two-tailed) using $\mathrm{G}^{*}$ Power (Faul, Erdfelder, Buchner, \& Lang, 2013).

All participants had normal or corrected-to-normal visual acuity and signed consent forms before participating in the study. All participants were Koreans and highly familiar with the Korean number system since childhood. The study was approved by the Seoul National University IRB.

\section{Stimuli and apparatus}

Two Korean number words or Arabic digits from 1 to 9 were presented side-by-side in black on a white square measuring $2.6 \mathrm{~cm}\left(2.48^{\circ}\right)$ by $2.6 \mathrm{~cm}\left(2.48^{\circ}\right)$; the remaining background was gray. Digits or words were simultaneously presented in pairs on the screen. Pairs containing the same word or digit were removed from the whole set of stimuli to ensure that different combinatorial pairs of stimuli were used. Accordingly, there were $72(9 \times 8)$ pairs under both the Korean word condition and the Arabic numeral condition. Both types of notations were presented in the same font (D2Coding 1.1), and their physical sizes were $2.1 \mathrm{~cm}\left(2.02^{\circ}\right)$ in width and $2.2 \mathrm{~cm}\left(2.15^{\circ}\right)$ in height for the Korean number words and $0.9 \mathrm{~cm}\left(0.86^{\circ}\right)$ in width and $1.4 \mathrm{~cm}\left(1.32^{\circ}\right)$ in height for the Arabic numerals. In each pair of stimuli, the two notations were presented $8 \mathrm{~cm}\left(7.62^{\circ}\right)$ apart from the center of the screen. The stimulus presentation and response input used E-Prime 2 (Psychology Software Tools, Pittsburgh, Pa.). Figure 2 (a) and (b) show the Korean and Arabic numeral stimuli, and (c) illustrates some example pairs.

Based on the congruency of the Korean number words, each pair of Korean number words or Arabic numeral stimuli was classified into one of three groups according to the number of letters constituting the number and the semantic size of the number. If the number of letters and the semantic size of the number matched, the pair was classified as congruent, but if they did not match, the pair was classified as incongruent; the remaining pairs were classified as neutral. Finally, in both the left and the right locations, the congruent, neutral, and incongruent conditions included 10, 16, and 10 pairs, respectively. Figure 3 shows the three types of classification of all 36 pairs.

\section{Design and procedures}

All participants performed the Arabic numeral condition task first and the Korean number condition task second. The observation distance from the monitor was approximately $60 \mathrm{~cm}$. In each task, the participant stared at the cross-shaped anchor point for $500 \mathrm{~ms}$, and then two Arabic digits or Korean number words were presented simultaneously. The participants were asked to choose the number on the screen with the larger meaning. If the number on the left was larger, the "q" key, allocated to the left index finger, was used. If the number on the right was larger, the " $p$ " key, assigned to the right index finger, was used. The stimulus remained on the screen until the response. Immediately after the response, the fixation points appeared, and the following trial was conducted. One task block consisted of a total of 72 left-hand and right-hand numerical pairs, which were presented randomly; each block was repeated four times. Therefore, each participant completed 576 trials as follows: 288 ( 36 pairs $\times 2$ position $\times 4$ repetitions) under the Arabic numeral condition and 288 (36 pairs $\times 2$ position $\times 4$ repetitions) under the Korean number condition. There were nine practice trials before each condition. Each experiment lasted approximately $35 \mathrm{~min}$.

\section{Results}

Before the analyses, the RTs of the incorrect responses were removed $(7.21 \%$ under the Korean number condition and $6.41 \%$ under the Arabic numeral condition). In addition, RT data greater than 3 standard deviations from the average RT of each participant were further excluded $(1.42 \%$ under the 


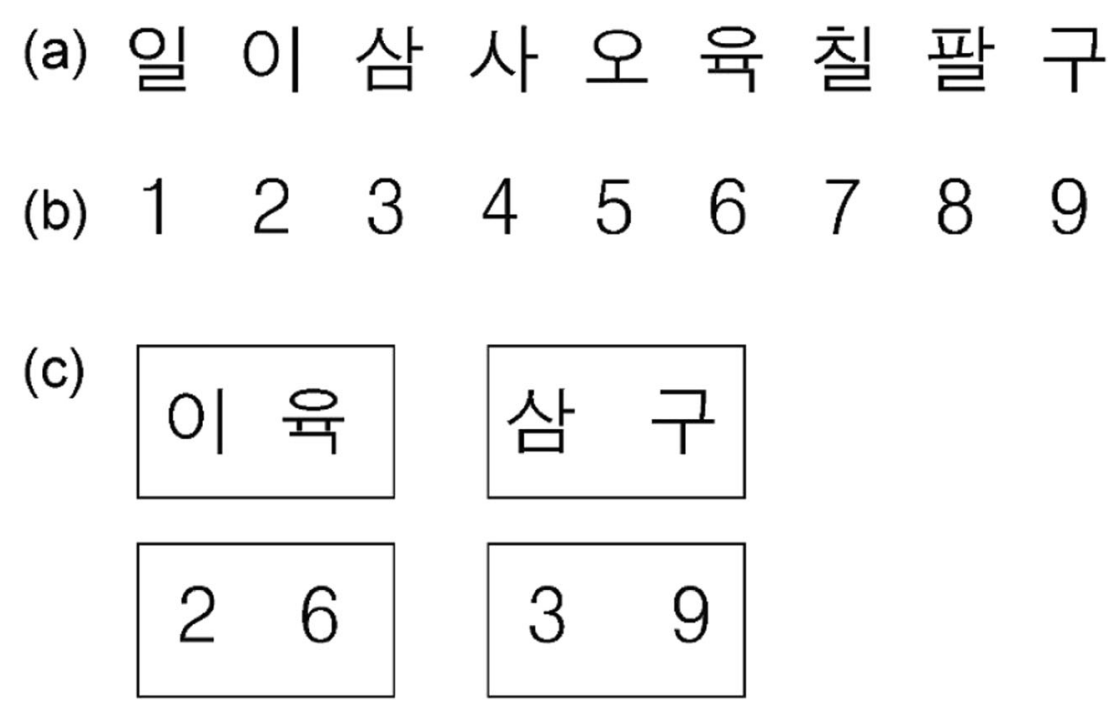

Fig. 2 Experimental stimuli and examples. a Korean number words. b Arabic numerals. The Arabic numerals in (b) correspond to the Korean words in (a). $\mathbf{c}$ The top row shows examples of the Korean congruent and

Korean number condition and $0.85 \%$ under the Arabic numeral condition).

Figure 4 shows the congruency effect between the semantic size of the two numbers and the number of letters based on the Korean number words. Notably, the congruency under the Arabic numeral condition was categorized based on the Korean number word pairs, which were identical to the Arabic digit pairs in semantic value. To examine how the notation method (Korean vs. Arabic) and congruency between the number of letters in each word and the number's semantic size influenced the number comparison process, a two-way repeated-measures ANOVA was performed. The analysis showed that under the Arabic numeral condition, the RT ( $M$ $=476.6 \mathrm{~ms}, S D=154.1$ ) was significantly faster than that under the Korean number word condition $(M=685.5 \mathrm{~ms}$, $S D=104.4), F(2,50)=38.86, p<.001, \eta_{p}{ }^{2}=.61$, supporting previous findings that the processing of Arabic numerals is generally faster than that of alphabetic number words (Schubert, 2017). The congruency effect was significant, incongruent conditions, and the bottom row shows the corresponding Arabic congruent and incongruent conditions

$F(2,50)=14.88, p<.001, \eta_{p}{ }^{2}=.37$ and faster (in descending order from fastest to slowest) as follows: congruent $(M=$ $568,1 \mathrm{~ms}, S D=165.5)$, neutral $(M=581.0 \mathrm{~ms}, S D=167.6)$, and incongruent $(M=593.9 \mathrm{~ms}, S D=172.8)$ conditions; congruent versus neutral: $t(25)=-2.733, p=.023$; neutral versus incongruent: $t(25)=-2.722, p=.023$; and congruent versus incongruent, $t(25)=-5.455, p<.001$. An interaction effect was found between the notation system of numbers and congruency, $F(2,50)=36.27, p<.001, \eta_{p}{ }^{2}=.59$. This result indicates that the congruency effect appears differently based on the notation system. Under the Korean word condition, the RT was the fastest under the congruent $(M=650.3 \mathrm{~ms}, S D=$ 112.6) condition, followed by the neutral $(M=689.4 \mathrm{~ms}, S D=$ $100.3)$ and incongruent $(M=716.6 \mathrm{~ms}, S D=92.3)$ conditions, $F(2,50)=36.67, p<.001, \eta_{p}{ }^{2}=.59$. The post hoc pairedsample $t$-tests indicated that all conditions differed as follows: congruent vs. neutral: $t(25)=-5.023, p<.001$; neutral versus incongruent: $t(25)=-3.496, p=.002$; and congruent versus incongruent: $t(25)=-8.518, p<.001$. In contrast, under the

$$
\begin{aligned}
& \begin{array}{ccccccccccccccccc}
\begin{array}{c}
\text { Congruent } \\
(n=10)
\end{array} & \text { 이 삼 } & \text { 이 육 } & \text { 이 칠 } & \text { 이 팔 } & \text { 사 육 } & \text { 사 칠 } & \text { 사 팔 } & \text { 오 육 } & \text { 오 칠 } & \text { 오 팔 } \\
\hline
\end{array}
\end{aligned}
$$

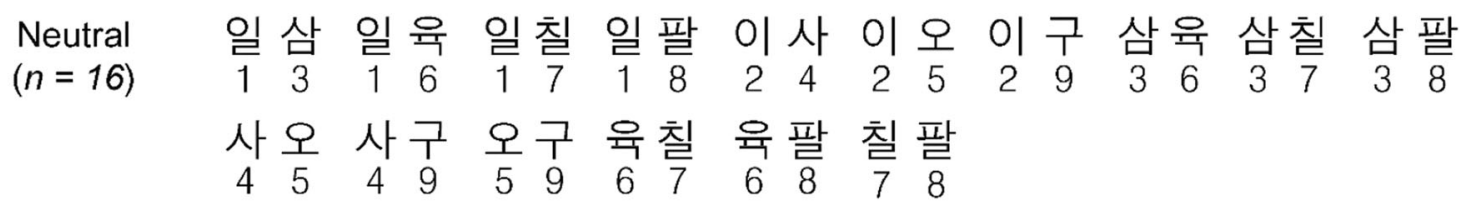

$$
\begin{aligned}
& \text { Incongruent 일 이 일 사 일오 일구 삼사 삼오 삼구 육구 칠구 팔구 } \\
& \begin{array}{lllllllllllllllllllll}
(n=10) & 1 & 2 & 1 & 4 & 1 & 5 & 1 & 9 & 3 & 4 & 3 & 5 & 3 & 9 & 6 & 9 & 7 & 9 & 8 & 9
\end{array}
\end{aligned}
$$

Fig. 3 Three types of conditions according to congruency and all possible number combinations. The top rows of each condition show the Korean number words, and the bottom rows show their corresponding Arabic numerals 


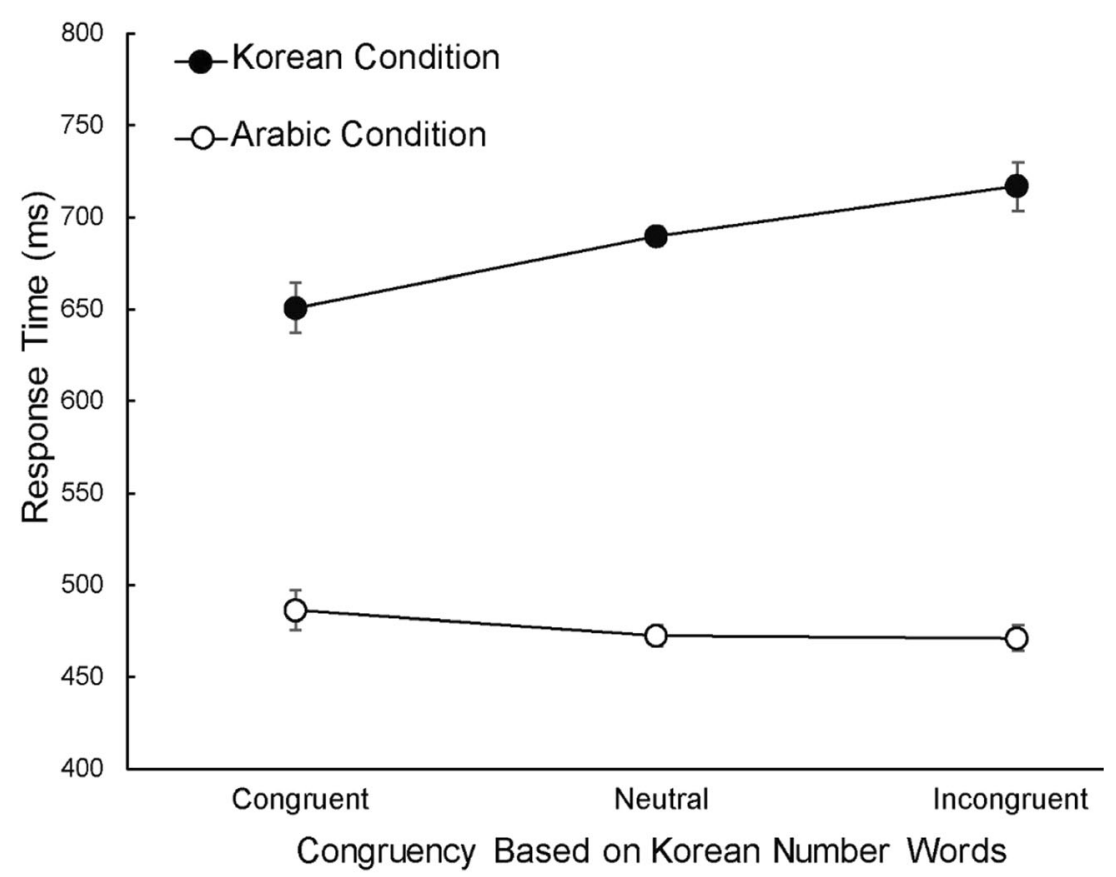

Fig. 4 Mean response times of the correct responses ranked by congruency. The congruency of the Arabic trials was determined by the Korean trials with corresponding semantic values. The error bars represent the within-subjects $95 \%$ confidence intervals

Arabic condition, the RT tended to become slower with congruency as follows: congruent $(M=485.9 \mathrm{~ms}, S D=170.7)$, neutral $(M=472.6, S D=150.9)$, and incongruent $(M=471.1$ $\mathrm{ms}, S D=145.1)$ conditions, $F(2,50)=4.33, p=.04, \eta_{p}{ }^{2}=.15$. The post hoc paired-sample $t$-tests indicated that the other conditions differed as follows: congruent versus neutral: $t(25)=2.401, p=.051$, and congruent versus incongruent: $t(25)=2.673, p=.026$. Although this result contradicts the result obtained under the Korean word condition, significant results were not expected. A possible explanation for this result may be found in the end effect (Leth-Steensen \& Marley, 2000; Verguts \& Van Opstal, 2014). Specifically, in the pairs containing a 1 or a 9 , the participants could respond correctly by processing only this digit without viewing the other number, thereby potentially reducing the RT. In fact, in this study, the average $\mathrm{RT}$ in response to the number pairs containing the Arabic digit 1 or 9 was faster $(M=459.2 \mathrm{~ms}, S D=140.1)$ than that in response to the other number pairs $(M=488.5 \mathrm{~ms}, S D$ $=165.9$ ). As shown in Fig. 3, the percentage of pairs containing a 1 or a 9 was high under the incongruent condition $(8 / 10$ cases $=80 \%)$ and lower under the neutral condition $(7 / 16$ cases $=43.7 \%$ ), while no such pairs were presented under the congruent condition $(0 / 10$ cases $=0 \%)$; these differences might explain the significant results across the three conditions using Arabic numerals.

To determine whether the number distance influenced the RT, the distance variable used the Welford function instead of the absolute value of the difference of the two numbers (Moyer \& Landauer, 1967) as follows: Response Time $=\log$ (larger number/larger number - smaller number). For the statistical testing, we used the Lorch and Myers method (1990, Method 3). This method calculates the regression coefficient of the independent variable for each individual set of RTs. As shown in Fig. 5a, the larger the distance between the two numbers under both the Korean and Arabic numeral conditions, the faster the RT, which is consistent with previous studies (Moyer \& Landauer, 1967). The analysis showed that the distance under the Korean condition (mean slope $=$ $103.710, t(25)=11.109, p<.001, d=2.17)$ and Arabic condition (mean slope $=104.182, t(25)=5.879, p<.001, d=$ 1.15 ) was a significant predictive variable, indicating that both notations automatically activate the semantic size of the number.

It could be argued that the congruency effect of the Korean condition might result from the distance effect. However, as shown in Fig. 3, the numerical difference is the largest under the neutral condition $(M=3.6, S D=2.06)$ and similar between the congruent condition and incongruent condition $(M=3.1$, $S D=1.66$ and $M=3.1, S D=2.33$, respectively). Additionally, a one-way repeated-measures ANCOVA was performed to statistically determine whether the congruency effect existed independently of the distance effect. Thus, the congruency effect was calculated using the following function: the number of letters in the Korean number word with a larger semantic size - the number of letters in the Korean number word with a smaller semantic size. Accordingly, the congruent, neutral and incongruent conditions were coded as " +1 ", " 0 ", and " -1 ", respectively. As a result, after statistically controlling for the effect of the numerical difference, the congruency effect was still significant, $F(2,50)=36.67, p<.001, \eta_{p}{ }^{2}=.59$. In 
(a)

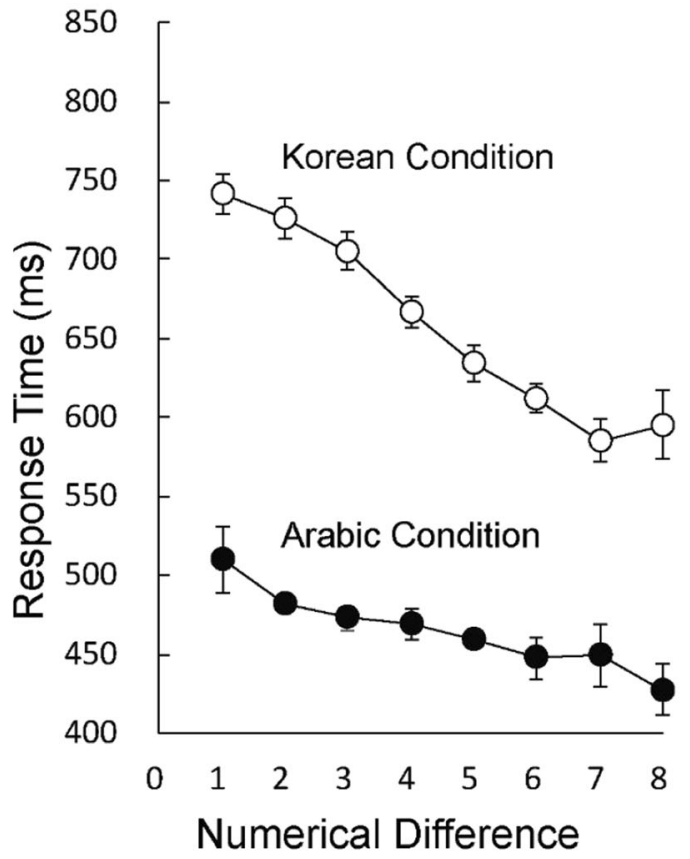

Fig. 5 Mean response times as a function of the numerical difference. a The data are categorized by Arabic numeral condition and Korean number word condition. The error bars represent the within-subjects 95\% confidence intervals. b The mean response times under the Arabic

summary, the congruency effect is presumed to be due to the number of letters rather than the numerical difference across numbers. As shown in Fig. 5b, the congruency and distance seem to influence the RT additively and independently.

\section{Discussion}

The effect of the number of letters constituting number words on numerical comparisons found in the present study is comparable with the continuous size congruency effect widely reported in previous studies. Considering that the participants were highly familiar with the number words' semantic values in relation to their figural forms, the congruency effect exerted by the number of letters suggests that the processing of the discrete properties of number notations is deeply rooted in the representation of the numbers' semantic size. Accordingly, we suggest that both the continuous properties and discrete properties of number notations are crucial for numerical representation. This paired role of the two forms of properties in numerical representation has been extensively tested using symbolic geometric stimuli, such as dots or lines. The present study suggests that such a role also exists in the processing of symbolic stimuli.

The continuous size congruency effect has been shown to depend on the phonological transparency between printed number notations and their pronunciations. Most evidence of (b)

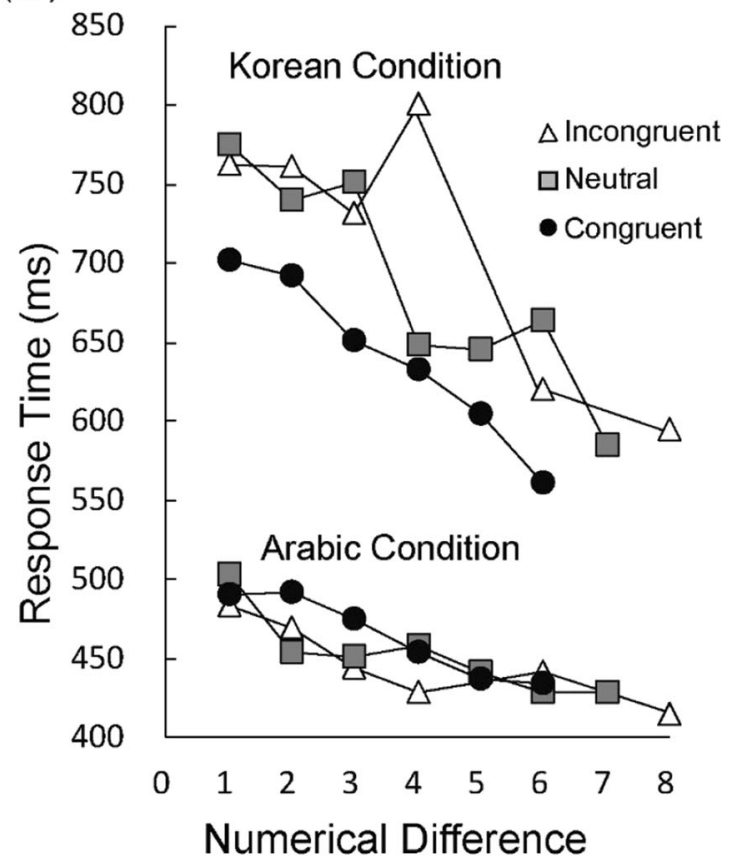

condition and Korean number word condition are separated based on the congruency between the number of letters in the Korean number words and the semantic size of the words

the continuous size congruency effect is observed with phonologically opaque scripts, such as Arabic numerals or English number words. In contrast, investigators did not find a size congruency effect with phonologically transparent scripts, such as Hindi scripts (Vaid, 1985), Japanese Kana scripts (Takahashi \& Green, 1983), and Hebrew letters (RazpurkerApfeld \& Koriat, 2006). Accordingly, investigators generally assume that when number notations are phonologically opaque, the visual route becomes important for reaching a magnitude estimation process, i.e., people are easily susceptible to perceptual properties, while when number notations are phonologically transparent, the phonological route mainly contributes to activating the process, i.e., people are less sensitive to perceptual properties (Razpurker-Apfeld \& Koriat, 2006; Vaid, 1985). In this case, how can the general assumption explain the discrete size congruency effect found in the present study? According to the assumption, because Korean scripts are phonologically highly transparent, i.e., a perceptual property, the number of letters in number words was not expected to influence the RT of the numerical comparison task. However, the results differ from this expectation. One compromising, tentative explanation may be that when people view number notations, the size representation of both the continuous and discrete perceptual dimensions of the notations is likely simultaneously activated, but the discrete dimension may more sensitively interact with the semantic size than the continuous dimension regardless of the phonological 
transparency. Additional studies are needed to examine this hypothesis in the future.

In a previous series of studies using multiple number notations, a discrete size congruency effect was reported (Naparstek \& Henik, 2010; Pansky \& Algom, 2002; Pavese \& Umiltà, 1998). Our results are largely commensurate with these findings. However, in those studies, multiple number notations were presented with fillers, such as "*” or alphabetic symbols, in circular or rectangular shaped displays. Accordingly, in those displays, at some point during the visual process, the number notations should be grouped together but segregated from the fillers. Thus, the number-occupied area paradox appears again as described in the Introduction section; therefore, whether the discrete size congruency effect is caused purely by the number of number notations or the occupied area is unclear. Considering this problem, our finding seems to more clearly show how the discrete dimension of written number notations interacts with their semantic size.

Acknowledgements We would like to thank Craig Leth-Steensen, Todd Kahan, and an anonymous reviewer for providing very supportive comments and suggestions regarding earlier drafts of the paper.

This work was supported by the Korea government (2019-0-01367BabyMind).

Open practices statement All data are available at https://osf.io/x3y2h.

\section{References}

Besner, D., \& Coltheart, M. (1979). Ideographic and alphabetic processing in skilled reading of English. Neuropsychologia, 17(5), 467-472.

Cohen Kadosh, R., Gevers, W., \& Notebaert, W. (2011). Sequential analysis of the numerical Stroop effect reveals response suppression. Journal of Experimental Psychology: Learning, Memory, and Cognition, 37(5), 1243-1249.

Damian, M. F. (2004). Asymmetries in the processing of Arabic digits and number words. Memory \& Cognition, 32(1), 164-171.

Dehaene, S., \& Akhavein, R. (1995). Attention, automaticity, and levels of representation in number processing. Journal of Experimental Psychology: Learning, Memory, and Cognition, 21(2), 314-326.

Dixon, P. (1978). Numerical comparison processes. Memory \& Cognition, 6(4), 454-461.

Dormal, V., Seron, X., \& Pesenti, M. (2006). Numerosity-duration interference: A Stroop experiment. Acta Psychologica, 121(2), 109-124.

Faul, F., Erdfelder, E., Buchner, A., \& Lang, A.-G. (2009). Statistical power analyses using $\mathrm{G}^{*}$ Power 3.1: Tests for correlation and regression analyses. Behavior Research Methods, 41, 1149-1160.

Feigenson, L., Dehaene, S., \& Spelke, E. (2004). Core systems of number. Trends in Cognitive Sciences, 8(7), 307-314.

Foltz, G. S., Poltrock, S. E., \& Potts, G. R. (1984). Mental comparison of size and magnitude: Size congruity effects. Journal of Experimental Psychology: Learning, Memory, and Cognition, 10(3), 442-453.
Henik, A., Leibovich, T., Naparstek, S., Diesendruck, L., \& Rubinsten, O. (2012). Quantities, amounts, and the numerical core system. Frontiers in Human Neuroscience, 5(186), 1-4.

Henik, A., \& Tzelgov, J. (1982). Is three greater than five: The relation between physical and semantic size in comparison tasks. Memory \& Cognition, 10(4), 389-395.

Ito, Y., \& Hatta, T. (2003). Semantic processing of Arabic, Kanji, and Kana numbers: Evidence from interference in physical and numerical size judgments. Memory \& Cognition, 31(3), 360-368.

Leibovich, T., \& Henik, A. (2013). Magnitude processing in nonsymbolic stimuli. Frontiers in Psychology, 4(375), 1-6.

Leth-Steensen, C., \& Marley, A. A. J. (2000). A model of response time effects in symbolic comparison. Psychological Review, 107(1), 62100.

Lorch, R. F., \& Myers, J. L. (1990). Regression analyses of repeated measures data in cognitive research. Journal of Experimental Psychology: Learning, Memory, and Cognition, 16(1), 149.

Mix, K. S., Huttenlocher, J., \& Levine, S. C. (2002). Multiple cues for quantification in infancy: Is number one of them? Psychological Bulletin, 128(2), 278-294.

Moyer, R. S., \& Landauer, T. K. (1967). Time required for judgements of numerical inequality. Nature, 215(5109), 1519-1520.

Naparstek, S., \& Henik, A. (2010). Count me in! On the automaticity of numerosity processing. Journal of Experimental Psychology: Learning, Memory, and Cognition, 36(4), 1053-1059.

Pansky, A., \& Algom, D. (2002). Comparative judgment of numerosity and numerical magnitude: Attention preempts automaticity. Journal of Experimental Psychology: Learning, Memory, and Cognition, $28(2), 259$.

Pavese, A., \& Umiltà, C. (1998). Symbolic distance between numerosity and identity modulates Stroop interference. Journal of Experimental Psychology: Human Perception and Performance, 24(5), 1535.

Razpurker-Apfeld, I., \& Koriat, A. (2006). Flexible mental processes in numerical size judgments: The case of Hebrew alphabets that are used to convey numbers. Psychonomic Bulletin \& Review, 13(1), 78-83.

Schubert, T. M. (2017). Why are digits easier to identify than alphabets? Neuropsychologia, 95, 136-155.

Schwarz, W., \& Heinze, H. J. (1998). On the interaction of numerical and size information in digit comparison: A behavioral and event-related potential study. Neuropsychologia, 36(11), 1167-1179.

Sobel, K. V., Puri, A. M., Faulkenberry, T. J., \& Dague, T. D. (2017). Visual search for conjunctions of physical and numerical size shows that they are processed independently. Journal of Experimental Psychology: Human Perception and Performance, 43(3), 444-453.

Takahashi, A., \& Green, D. (1983). Numerical judgments with Kanji and Kana. Neuropsychologia, 21(3), 259-263.

Tzeng, O. J., \& Wang, W. S.-Y. (1983). The first two R's: The way different languages reduce speech to script affects how visual information is processed in the brain. American Scientist, 71(3), 238-243.

Vaid, J. (1985). Numerical size comparisons in a phonologically transparent script. Perception \& Psychophysics, 37(6), 592-595.

Verguts, T., \& Van Opstal, F. (2014). A delta-rule model of numerical and non-numerical order processing. Journal of Experimental Psychology: Human Perception \& Performance, 40, 1092-1102.

Publisher's note Springer Nature remains neutral with regard to jurisdictional claims in published maps and institutional affiliations. 